\title{
The Vortex-like Behavior of the Riemann Zeta Function to the Right of the Critical Strip
}

\author{
J. M. Sepulcre@ and T. Vidal
}

\begin{abstract}
Based on an equivalence relation that was established recently on exponential sums, in this paper we study the class of functions that are equivalent to the Riemann zeta function in the half-plane $\{s \in \mathbb{C}$ : $\operatorname{Re} s>1\}$. In connection with this class of functions, we first determine the value of the maximum abscissa from which the images of any function in it cannot take a prefixed argument. The main result shows that each of these functions experiments a vortex-like behavior in the sense that the main argument of its images varies indefinitely near the vertical line $\operatorname{Re} s=1$. In particular, regarding the Riemann zeta function $\zeta(s)$, for every $\sigma_{0}>1$ we can assure the existence of a relatively dense set of real numbers $\left\{t_{m}\right\}_{m \geq 1}$ such that the parametrized curve traced by the points $\left(\operatorname{Re}\left(\zeta\left(\sigma+i t_{m}\right)\right), \operatorname{Im}\left(\zeta\left(\sigma+i t_{m}\right)\right)\right)$, with $\sigma \in\left(1, \sigma_{0}\right)$, makes a prefixed finite number of turns around the origin.
\end{abstract}

Mathematics Subject Classification. 11M06, 42A75, 30B50, 11K60, 30D20, 30Axx.

Keywords. Riemann zeta function, exponential sums, almost periodic functions, Bohr's equivalence relation, vortex-like behavior.

\section{Introduction}

Given a complex variable $s=\sigma+i t$, the exponential sums of the type

$$
\sum_{n \geq 1} a_{n} e^{-\lambda_{n} s}, a_{n} \in \mathbb{C},
$$

where $\left\{\lambda_{n}\right\}$ is a strictly increasing sequence of positive numbers tending to infinity, are known by the name of general Dirichlet series. A classical example 
is connected with the Riemann zeta function $\zeta(s)$, which is defined as the analytic continuation of the function defined for $\sigma>1$ by the sum $\sum_{n=1}^{\infty} \frac{1}{n^{s}}$. Any non-trivial zero of $\zeta(s)$ (i.e. different from $-2,-4, \ldots)$ lies in the open strip $\{s \in \mathbb{C}: 0<\operatorname{Re} s<1\}$, which is called the critical strip. It is widely known that the distribution of these zeros has captured the attention of many different researchers in view of the fact that their study plays a pivotal role in analytic number theory. In fact, it yields important results concerning prime numbers and related objects in number theory.

As a result of his investigations and contributions to the understanding of general Dirichlet series (and their regions of convergence, uniform convergence and absolute convergence), the Danish mathematical H. Bohr introduced an equivalence relation among them that led to important results in the last century. In particular, the so-called Bohr's equivalence theorem shows that equivalent Dirichlet series take the same values in certain vertical lines or strips in the complex plane (e.g. see $[1,2,6,11]$ ).

By using Bohr's theory as a starting point, we established in [7] a more general perspective through an equivalence relation $\sim$ on the classes $\mathcal{S}_{\Lambda}$ consisting of exponential sums of the form

$$
\sum_{j \geq 1} a_{j} e^{\lambda_{j} p}, a_{j} \in \mathbb{C}, \lambda_{j} \in \Lambda,
$$

where $\Lambda=\left\{\lambda_{1}, \lambda_{2}, \ldots, \lambda_{j}, \ldots\right\}$ is an arbitrary countable set of distinct real numbers, and $p$ is a parameter (in our case, it will be changed by $s=\sigma+$ it in the complex case, or by $t$ in the real case). In the context of almost periodic functions, to which this equivalence relation can also be extended, one of the main results of [7] shows that the condition of almost periodicity yields the fact that every sequence of (vertical) translates has a subsequence that converges uniformly (on every reduced strip) to an equivalent function (see also [8]). Furthermore, we extracted in [7, Section 5] some concrete applications to the case of exponential sums which converge absolutely, and in particular to the Riemann zeta function. For example, we show that any exponential sum which is equivalent to the Riemann zeta function, $\zeta(s)$, can be uniformly approximated in every reduced strip of $\{s \in \mathbb{C}: \operatorname{Re} s>1\}$ by certain vertical translates of $\zeta(s)$ (see [7, Theorem 6]). Throughout this work, we will use a generalization of Bohr's equivalence relation, defined in Sect. 2, which was used in [9] (see also [10]) to get a result like Bohr's equivalence theorem extended to certain classes of almost periodic functions in vertical strips $\{s \in \mathbb{C}: \alpha<$ $\operatorname{Re} s<\beta\}$.

Regarding the Riemann zeta function $\zeta(s)$, the connection between it and prime numbers was discovered by L. Euler, who proved the identity

$$
\zeta(s)=\prod_{k=1}^{\infty} \frac{1}{1-p_{k}^{-s}} \text { for } \operatorname{Re} s>1,
$$


where the product on the right hand extends over all prime numbers $p_{k}$. In view of the Euler product, it is easily seen that $\zeta(s)$ has no zeros in the halfplane $\{s \in \mathbb{C}: \operatorname{Re} s>1\}$. It is also known that the Dirichlet series and the Euler product of $\zeta(s)$ converge absolutely in the same half-plane $\sigma>1$ and uniformly in $\sigma \geq 1+\delta$ for any $\delta>0$. A more advanced introduction to the theory surrounding the Riemann hypothesis can be found for example in [3].

In general terms, let $\left\{a_{2}, a_{3}, a_{5}, \ldots, a_{p_{j}}, \ldots\right\}$ be an arbitrary sequence of complex numbers such that $\left|a_{p_{j}}\right|=1$ for each $j=1,2, \ldots$. Take $a_{1}=1$ and define $a_{n}=a_{p_{1}}^{\alpha_{1}} a_{p_{2}}^{\alpha_{2}} \cdots a_{p_{k_{n}}}^{\alpha_{k_{n}}}$ when $n=p_{1}^{\alpha_{1}} p_{2}^{\alpha_{2}} \cdots p_{k_{n}}^{\alpha_{k_{n}}}$ is not a prime number. Throughout this paper, associated with such a sequence $\left\{a_{2}, a_{3}, a_{5}, \ldots\right\}$, we will consider the generic exponential sum

$$
\sum_{n=1}^{\infty} \frac{a_{n}}{n^{s}}
$$

which also converges absolutely in $\sigma>1$ and uniformly in $\sigma \geq 1+\delta$ for any $\delta>0$. For example, the choices $a_{p_{j}}=1$ for each $j=1,2, \ldots$ and $a_{p_{j}}=$ -1 for each $j=1,2, \ldots$ provide respectively the Riemann zeta function and the Dirichlet series of the Liouville function $\lambda(n)=(-1)^{\Omega(n)}$, where $\Omega(n)$ is the number of prime factors of $n$ (counted with multiplicities). Precisely, we show in Proposition 3 that the image of these two functions on the real axis provides the above and below bounds for the absolute value of the image of each exponential sum of type (2) throughout every vertical line or closed half-plane in $\{s \in \mathbb{C}: \operatorname{Re} s>1\}$.

With respect to the arguments of all exponential sums of type (2), the special choices $a_{p_{j}}=i$ for each $j=1,2, \ldots$ and $a_{p_{j}}=-i$ for each $j=$ $1,2, \ldots$ provide bounds for the change of these arguments (see Lemma 4 ) and, in fact, they allows us to determine the maximum abscissa from which the images of any exponential sum of type (2) cannot take a prefixed argument (see Lemma 7). In particular, this yields that the images of any function equivalent to the Riemann zeta function cannot take negative real values on a certain half-plane of the form $\left\{s \in \mathbb{C}: \operatorname{Re} s>\sigma_{\pi}\right\}$, with $\sigma_{\pi}>1$.

Likewise, from Euler-type product formula for these sums $S(s)$ of type (2) (see Lemma 2), the main result of our paper shows that each one of these functions, and in particular the Riemann zeta function, experiments a vortexlike behavior in the sense that, given $\sigma_{0}>1$ and $n \in \mathbb{N}$, there exists a relatively dense set of real numbers $\left\{t_{n, m}\right\}_{m \geq 1}$ such that, for each $m=1,2, \ldots$, the image of the vector-valued function $\left(\operatorname{Re} S\left(\sigma+i t_{n, m}\right), \operatorname{Im} S\left(\sigma+i t_{n, m}\right)\right.$ ), for $\sigma$ in the interval $\left(1, \sigma_{0}\right)$, traces a curve in the plane which makes at least $n$ turns around the origin (see Theorem 11 in this paper, and related results in Lemma 5 and Propositions 9 and 10). To the best of our knowledge, this result has not been reported in the literature. 


\section{The Class of Functions Equivalent to the Riemann Zeta Function}

Based on the Bohr's equivalence relation, which was considered in [1, p. 173] for general Dirichlet series, we defined in [7-10] new equivalence relations in the more general context of the classes $S_{\Lambda}$ of exponential sums of type (1). In this paper, we will use the following definition which constitutes the same equivalence relation as that of $[9$, Definition 2].

Definition 1. Given $\Lambda=\left\{\lambda_{1}, \lambda_{2}, \ldots, \lambda_{j}, \ldots\right\}$ a set of distinct real numbers, consider $A_{1}(p)$ and $A_{2}(p)$ two exponential sums in the class $\mathcal{S}_{\Lambda}$, say $A_{1}(p)=$ $\sum_{j \geq 1} a_{j} e^{\lambda_{j} p}$ and $A_{2}(p)=\sum_{j \geq 1} b_{j} e^{\lambda_{j} p}$. We will say that $A_{1}(p)$ is equivalent to $A_{2}(p)$ if there exists a $\mathbb{Q}$-linear map $\psi: \operatorname{span}_{\mathbb{Q}}(\Lambda) \rightarrow \mathbb{R}$ such that $b_{j}=a_{j} e^{i \psi\left(\lambda_{j}\right)}$ for each $j=1,2, \ldots$.

Let $G_{\Lambda}=\left\{g_{1}, g_{2}, \ldots, g_{k}, \ldots\right\}$ be a basis of the vector space over the rational numbers generated by a set $\Lambda=\left\{\lambda_{1}, \lambda_{2}, \ldots, \lambda_{j}, \ldots\right\}$, which implies that $G_{\Lambda}$ is linearly independent over the rational numbers and each $\lambda_{j}$ is expressible as a finite linear combination of terms of $G_{\Lambda}$, say

$$
\lambda_{j}=\sum_{k=1}^{q_{j}} r_{j, k} g_{k}, \text { for some } r_{j, k} \in \mathbb{Q} .
$$

By abuse of notation, we will say that $G_{\Lambda}$ is a basis for $\Lambda$. Moreover, we will say that $G_{\Lambda}$ is an integral basis for $\Lambda$ when $r_{j, k} \in \mathbb{Z}$ for each $j, k$, i.e. $\Lambda \subset \operatorname{span}_{\mathbb{Z}}\left(G_{\Lambda}\right)$ (it is worth noting that all the results of [7] which can be formulated in terms of an integral basis are also valid under Definition 1 ).

In the particular case of the Riemann zeta function $\zeta(s)=\sum_{n>1} \frac{1}{n^{s}}$, with $\operatorname{Re} s>1$, we can take $\left\{\log 2, \log 3, \ldots, \log p_{k}, \ldots\right\}$, where $p_{k}$ is the $k$-th prime number, as an integral basis for the set $\Lambda$. Likewise, the set of exponential sums which are equivalent to $\zeta(s)$ are given by the series, for every choice of $\mathbf{x}=\left(x_{1}, x_{2}, \ldots, x_{k}, \ldots\right) \in \mathbb{R}^{\infty}$, of the form

$$
\zeta_{\mathbf{x}}(s):=\sum_{n \geq 1} e^{<\mathbf{r}_{n}, \mathbf{x}>i} n^{-s}, \text { with } \operatorname{Re} s>1,
$$

where $\mathbf{r}_{n}$ is the vector of integer components satisfying $\log n=<\mathbf{r}_{n}, \mathbf{g}>$ with $\mathbf{g}$ given by $\left(\log 2, \log 3, \ldots, \log p_{k}, \ldots\right)$ (see, for instance, [1, Section 8.8], [7, Proposition 1] or [9, Expression (2.2)]). In particular, the vector $\mathbf{x}=$ $(\pi, \pi, \ldots, \pi, \ldots)$ generates the Dirichlet series for the Liouville function, denoted here as $\zeta_{\pi}(s)$, which is related to the Riemann zeta function $[4,12]$ by

$$
\zeta_{\pi}(s)=\sum_{n=1}^{\infty} \frac{\lambda(n)}{n^{s}}=\frac{\zeta(2 s)}{\zeta(s)}, \text { for } \operatorname{Re} s>1,
$$

where $\lambda(n)$ is the Liouville's function. Hence $\zeta_{\boldsymbol{\pi}}(s)$ has no singular points in the domain $\operatorname{Re} s>1$. 
Now, let $\left\{a_{2}, a_{3}, a_{5}, \ldots, a_{p_{j}}, \ldots\right\}$ be an arbitrary sequence of complex numbers such that $\left|a_{p_{j}}\right|=1$ for each $j=1,2, \ldots$. Take $a_{1}=1$ and define $a_{n}=a_{p_{1}}^{\alpha_{1}} a_{p_{2}}^{\alpha_{2}} \cdots a_{p_{k_{n}}}^{\alpha_{k_{n}}}$ when $n=p_{1}^{\alpha_{1}} p_{2}^{\alpha_{2}} \cdots p_{k_{n}}^{\alpha_{k_{n}}}$ is not a prime number. Note that the exponential sum $\sum_{n>1} \frac{a_{n}}{n^{s}}$ is identified with that of (3) given by $\zeta_{\mathbf{x}}(s)=\sum_{n>1} \frac{e^{<\mathbf{r}_{n}, \mathbf{x}>i}}{n^{s}}$, where $\mathbf{x}=\left(x_{1}, x_{2}, \ldots, x_{k}, \ldots\right)$ satisfies $a_{p_{j}}=e^{i x_{j}}$ for each $j=1,2, \ldots$. Indeed, we have that

$$
a_{n}=a_{p_{1}}^{\alpha_{1}} a_{p_{2}}^{\alpha_{2}} \cdots a_{p_{k_{n}}}^{\alpha_{k_{n}}}=e^{i\left(\alpha_{1} x_{1}+\alpha_{2} x_{2}+\ldots+\alpha_{k_{n}} x_{k_{n}}\right)}=e^{<\mathbf{r}_{n}, \mathbf{x}>i},
$$

where $\mathbf{r}_{n}$ is defined above. Consequently, we will handle these series written in the form of (3).

Although the following preliminary results are reasonably simple, we next provide their proof for the sake of completeness. We first obtain an Euler-type product for $\zeta_{\mathbf{x}}(s)$ on $\operatorname{Re} s>1$ and we prove that the convergence of this Eulertype product is uniform in every half-plane $\operatorname{Re} s \geq 1+\delta, \delta>0$.

Lemma 2. Given $\mathbf{x}=\left(x_{1}, x_{2}, \ldots, x_{k}, \ldots\right) \in \mathbb{R}^{\infty}$, the product $\prod_{k=1}^{\infty} \frac{1}{1-e^{i x_{k}} p_{k}^{-s}}$ converges uniformly to $\zeta_{\mathbf{x}}(s)$ in every half-plane $\{s \in \mathbb{C}: \operatorname{Re} s \geq 1+\delta\}$, with $\delta>0$.

Proof. Since $\frac{1}{2^{s}} \zeta_{\mathbf{x}}(s)=\sum_{n=1}^{\infty} \frac{e^{<\mathbf{r}_{n}, \mathbf{x}>i}}{(2 n)^{s}}$ for any $s$ with $\operatorname{Re} s>1$, the function $\zeta_{\mathbf{x}}(s)$ satisfies

$$
\zeta_{\mathbf{x}}(s)\left(1-e^{i x_{1}} 2^{-s}\right)=1+\frac{e^{i x_{2}}}{3^{s}}+\frac{e^{i x_{3}}}{5^{s}}+\frac{e^{i x_{4}}}{7^{s}}+\frac{e^{2 i x_{2}}}{9^{s}}+\frac{e^{i x_{5}}}{11^{s}}+\ldots
$$

Analogously,

$$
\zeta_{\mathbf{x}}(s)\left(1-e^{i x_{1}} 2^{-s}\right)\left(1-e^{i x_{2}} 3^{-s}\right)=1+\frac{e^{i x_{3}}}{5^{s}}+\frac{e^{i x_{4}}}{7^{s}}+\frac{e^{i x_{5}}}{11^{s}}+\ldots
$$

and, in general, for the first $m$ primes we have

$$
\zeta_{\mathbf{x}}(s) \prod_{k=1}^{m}\left(1-e^{i x_{k}} p_{k}^{-s}\right)=1+\frac{e^{<\mathbf{r}_{l_{1}}, \mathbf{x}>i}}{l_{1}^{s}}+\frac{e^{<\mathbf{r}_{l_{2}}, \mathbf{x}>i}}{l_{2}^{s}}+\ldots
$$

where $l_{1}, l_{2}, \ldots$ are the natural numbers which are not divisible by any of the $m$ first prime numbers, and hence $\left\{l_{1}, l_{2}, \ldots\right\} \subset\left\{p_{m+1}, p_{m+1}+1, \ldots\right\}$. In this way, given $\delta>0$, for any $s=\sigma+i t$ such that $\sigma \geq 1+\delta$ we get 


$$
\begin{aligned}
& \left|\zeta_{\mathbf{x}}(s)-\prod_{k=1}^{m} \frac{1}{1-e^{i x_{k}} p_{k}^{-s}}\right|=\left|\prod_{k=1}^{m} \frac{1}{1-e^{i x_{k}} p_{k}^{-s}}\right|\left|\zeta_{\mathbf{x}}(s) \prod_{k=1}^{m}\left(1-e^{i x_{k}} p_{k}^{-s}\right)-1\right| \\
& \quad=\prod_{k=1}^{m} \frac{1}{\left|1-e^{i x_{k}} p_{k}^{-s}\right|}\left|\frac{e^{<\mathbf{r}_{l_{1}}, \mathbf{x}>i}}{l_{1}^{s}}+\frac{e^{<\mathbf{r}_{l_{2}}, \mathbf{x}>i}}{l_{2}^{s}}+\ldots\right| \\
& \quad \leq \prod_{k=1}^{m} \frac{1}{1-p_{k}^{-\sigma}}\left(\frac{1}{l_{1}^{\sigma}}+\frac{1}{l_{2}^{\sigma}}+\ldots\right) \leq \zeta(1+\delta) \sum_{k=p_{m+1}}^{\infty} \frac{1}{k^{1+\delta}},
\end{aligned}
$$

which tends to 0 as $m \rightarrow \infty$. Hence the result holds.

The following result, which is a clear consequence of the Euler product representation, shows that the functions $\zeta_{\mathbf{x}}(s)$ are bounded throughout every vertical line $\left\{s \in \mathbb{C}: \operatorname{Re} s=\sigma_{0}\right\}$ or closed half-plane $\left\{s \in \mathbb{C}: \operatorname{Re} s \geq \sigma_{0}\right\}$ included in $\{s \in \mathbb{C}: \operatorname{Re} s>1\}$.

Proposition 3. Let $\mathbf{x}=\left(x_{1}, x_{2}, \ldots, x_{k}, \ldots\right) \in \mathbb{R}^{\infty}$ and $K$ be a closed half-plane in $\{s \in \mathbb{C}: \operatorname{Re} s>1\}$. Then

$$
\zeta_{\pi}\left(\sigma_{0}\right) \leq\left|\zeta_{\mathbf{x}}(s)\right| \leq \zeta\left(\sigma_{0}\right) \text { for any } s \in K,
$$

where $\sigma_{0}=\min \{\operatorname{Re} s: s \in K\}$.

Proof. Let $K$ be a closed half-plane in $\{s \in \mathbb{C}: \operatorname{Re} s>1\}$, and take the value $\sigma_{0}=\min \{\operatorname{Re} s: s \in K\}$ (which is greater than 1). Take $s=\sigma+i t \in K$. By the Euler-type product formula, we have

$$
\left|\zeta_{\mathbf{x}}(\sigma+i t)\right|=\left|\prod_{k \geq 1} \frac{1}{1-e^{i x_{k}} p_{k}^{-\sigma-i t}}\right| \geq \prod_{k \geq 1} \frac{1}{1+p_{k}^{-\sigma}} \geq \prod_{k \geq 1} \frac{1}{1+p_{k}^{-\sigma_{0}}}=\zeta_{\pi}\left(\sigma_{0}\right) .
$$

Moreover, it is clear that

$$
\left|\zeta_{\mathbf{x}}(s)\right|=\left|\sum_{n \geq 1} \frac{e^{<\mathbf{r}_{n}, \mathbf{x}>i}}{n^{\sigma+i t}}\right| \leq \sum_{n \geq 1} \frac{1}{n^{\sigma}} \leq \sum_{n \geq 1} \frac{1}{n^{\sigma_{0}}}=\zeta\left(\sigma_{0}\right) .
$$

Thus the result holds.

\section{On the Values of the Arguments of the Functions that are Equivalent to the Riemann Zeta Function}

Let $\zeta_{\mathbf{x}}(s)=\sum_{n>1} e^{<\mathbf{r}_{j}, \mathbf{x}>i} e^{-s \log n}$, with $\operatorname{Re} s>1$ and $\mathbf{x} \in \mathbb{R}^{\infty}$, be an exponential sum which is equivalent to the Riemann zeta function. By Lemma 2 we know that $\zeta_{\mathbf{x}}(s)$ can be expressed in terms of the Euler-type product $\prod_{k=1}^{\infty} \frac{1}{1-e^{i x_{k}} p_{k}^{-s}}$ and this product converges uniformly to $\zeta_{\mathbf{x}}(s)$ in every 
reduced strip of $U=\{s \in \mathbb{C}: \operatorname{Re} s>1\}$. This yields that the principal value of the argument of $\zeta_{\mathbf{x}}(s)$ can be written in terms of

$$
\operatorname{Arg}\left(\zeta_{\mathbf{x}}(s)\right)=-\sum_{k \geq 1} \operatorname{Arg}\left(1-e^{i x_{k}} p_{k}^{-s}\right)(\bmod (-\pi, \pi]) .
$$

From now on, we will denote as Args the principal value of the argument of a non-null complex value $s$. In our case, given $\mathbf{x} \in \mathbb{R}^{\infty}$, we will handle the mapping $A_{\zeta_{\mathbf{x}}}(s): U \mapsto \mathbb{R}_{+} \cup\{\infty\}$ defined as

$$
A_{\zeta_{\mathbf{x}}}(s):=\sum_{k \geq 1}\left|\operatorname{Arg}\left(1-e^{i x_{k}} p_{k}^{-s}\right)\right|
$$

Notice that $\lim _{\sigma \rightarrow \infty} \zeta(\sigma+i t)=1$ for any $t \in \mathbb{R}$ (and hence $\lim _{\sigma \rightarrow \infty} \operatorname{Arg}(\zeta(\sigma+$ $i t))=0$ for any $t \in \mathbb{R})$. So, thanks to $[9$, Theorem 18], we state that every function $\zeta_{\mathbf{x}}(s)$ satisfies that $\lim _{\sigma \rightarrow \infty} \zeta_{\mathbf{x}}(\sigma+i t)=1$ for any $t \in \mathbb{R}$ (and hence $\lim _{\sigma \rightarrow \infty} \operatorname{Arg}\left(\zeta_{\mathbf{x}}(\sigma+i t)\right)=0$ for any $\left.t \in \mathbb{R}\right)$. In particular, we have that $\lim _{\sigma \rightarrow \infty} A_{\zeta_{\mathbf{x}}}(\sigma+i t)=0$ for any $t \in \mathbb{R}$.

The following lemma shows the importance of $\zeta_{\frac{\pi}{2}}(s)=\prod_{k=1}^{\infty} \frac{1}{1-i p_{k}^{-s}}$ and $\zeta_{-\frac{\pi}{2}}(s)=\prod_{k=1}^{\infty} \frac{1}{1+i p_{k}^{-s}}$ in terms of the mapping $A_{\zeta_{\mathbf{x}}}(s)$, which is connected with the argument of the functions $\zeta_{\mathbf{x}}(s)$ equivalent to the Riemann zeta function.

Lemma 4. Let $A_{\zeta_{\mathbf{x}}}(s)=\sum_{k \geq 1}\left|\operatorname{Arg}\left(1-e^{i x_{k}} p_{k}^{-s}\right)\right|$ be the mapping defined above. It is satisfied:

(i) $A_{\zeta_{\mathbf{x}}}(\sigma+i t) \leq A_{\zeta_{\frac{\pi}{2}}}(\sigma)=A_{\zeta_{-\frac{\pi}{2}}}(\sigma)$ for any $\mathbf{x} \in \mathbb{R}^{\infty}$ and $\sigma+i t \in U$.

(ii) $A_{\zeta \frac{\pi}{2}}(\sigma)=\sum_{k \geq 1} \arctan \left(p_{k}^{-\sigma}\right)$ for any $\sigma>1$;

(iii) $A_{\zeta_{\frac{\pi}{2}}}(\sigma)<\infty$ for any $\sigma>1$, and $\lim _{\sigma \rightarrow 1^{+}} A_{\zeta_{\frac{\pi}{2}}}(\sigma)=\infty$;

(iv) The function $A_{\zeta_{\frac{\pi}{2}}}:(1, \infty) \mapsto \mathbb{R}_{+}$, defined as

$$
A_{\zeta_{\frac{\pi}{2}}}(\sigma)=\sum_{k \geq 1}\left|\operatorname{Arg}\left(1-i p_{k}^{-\sigma}\right)\right|
$$

is continuous and decreasing.

Proof. (i) Given $\sigma>1$ and $k \in \mathbb{N}$, it is clear that $\left|\operatorname{Arg}\left(1-e^{i x_{k}} p_{k}^{-\sigma}\right)\right|$ attains the maximum value when $x_{k}= \pm \frac{\pi}{2}$. In the same way, fixed $\sigma+i t \in \mathbb{C}$ with $\sigma>1$, we have $\left|\operatorname{Arg}\left(1-e^{i x_{k}} p_{k}^{-\sigma-i t}\right)\right|=\left|\operatorname{Arg}\left(1-e^{i\left(x_{k}-t \log p_{k}\right)} p_{k}^{-\sigma}\right)\right| \leq$ $\left|\operatorname{Arg}\left(1+i p_{k}^{-\sigma}\right)\right|=\left|\operatorname{Arg}\left(1-i p_{k}^{-\sigma}\right)\right|$ for each $k=1,2, \ldots$, which proves (i).

(ii) Given $\sigma>1$, note that

$$
\operatorname{Arg}\left(1-i p_{k}^{-\sigma}\right)=\arctan \left(-p_{k}^{-\sigma}\right)=-\arctan \left(p_{k}^{-\sigma}\right)
$$

and

$$
\operatorname{Arg}\left(1+i p_{k}^{-\sigma}\right)=\arctan \left(p_{k}^{-\sigma}\right)
$$


Hence

$$
A_{\zeta_{ \pm \frac{\pi}{2}}}(\sigma)=\sum_{k \geq 1}\left|\arctan \left(p_{k}^{-\sigma}\right)\right|=\sum_{k \geq 1} \arctan \left(p_{k}^{-\sigma}\right)
$$

(iii) For $\sigma>0$, it is easy to prove that $\frac{3 \sigma}{3+\sigma^{2}}<\arctan \sigma<\sigma$ (see for example [5, p. 665] for the first inequality). Hence

$$
\sum_{k \geq 1} \frac{3 p_{k}^{-\sigma}}{3+p_{k}^{-2 \sigma}} \leq \sum_{k \geq 1} \arctan \left(p_{k}^{-\sigma}\right) \leq \sum_{k \geq 1} p_{k}^{-\sigma}
$$

Moreover, we have that $\sum_{k \geq 1} \frac{1}{p_{k}^{\sigma}}$ and $\sum_{k \geq 1} \frac{3 / p_{k}^{\sigma}}{3+1 / p_{k}^{2 \sigma}}$ have the same character of convergence. Consequently,

$$
\sum_{k \geq 1} \arctan \left(p_{k}^{-\sigma}\right)=A_{\zeta \frac{\pi}{2}}(\sigma)<\infty \text { for any } \sigma>1
$$

and

$$
\lim _{\sigma \rightarrow 1^{+}} \sum_{k \geq 1} \arctan \left(p_{k}^{-\sigma}\right)=\lim _{\sigma \rightarrow 1^{+}} A_{\zeta_{\frac{\pi}{2}}}(\sigma)=\infty .
$$
then

(iv) It is clear that $\lim _{\sigma \rightarrow \infty} A_{\zeta_{\frac{\pi}{2}}}(\sigma)=0$. Furthermore, if $1<\sigma_{1}<\sigma_{2}$

$$
\left|\operatorname{Arg}\left(1-i p_{k}^{-\sigma_{1}}\right)\right|>\left|\operatorname{Arg}\left(1-i p_{k}^{-\sigma_{2}}\right)\right| \text { for each } k=1,2, \ldots
$$

Consequently,

$$
A_{\zeta_{\frac{\pi}{2}}}\left(\sigma_{1}\right)=\sum_{k \geq 1}\left|\operatorname{Arg}\left(1-i p_{k}^{-\sigma_{1}}\right)\right| \geq \sum_{k \geq 1}\left|\operatorname{Arg}\left(1-i p_{k}^{-\sigma_{2}}\right)\right|=A_{\zeta_{\frac{\pi}{2}}}\left(\sigma_{2}\right),
$$

which proves that $A_{\zeta_{\frac{\pi}{2}}}(\sigma)$ is decreasing on $(1, \infty)$. Moreover, by Weierstrass's criterion, the sum

$$
\sum_{k \geq 1}\left|\operatorname{Arg}\left(1-i p_{k}^{-\sigma}\right)\right|=\sum_{k \geq 1} \arctan \left(p_{k}^{-\sigma}\right) \leq \sum_{k \geq 1} p_{k}^{-\sigma}
$$

converges uniformly on every reduced strip of $U$, which proves the continuity of $A_{\zeta \frac{\pi}{2}}(\sigma)$.

As a consequence of the lemma above, given $\mathbf{x} \in \mathbb{R}^{\infty}$, the mapping $A_{\zeta_{\mathbf{x}}}(s): U \mapsto \mathbb{R}_{+}$considered in (4) leads to a function well defined. Furthermore, it is clear that the case $\zeta_{\frac{\pi}{2}}(s)$ is particularly significant in our context. We next prove the following lemma regarding this function.

Lemma 5. Fixed $\varepsilon>0$, the parametrized curve traced by the points $\left(\operatorname{Re}\left(\zeta_{\frac{\pi}{2}}(\sigma)\right)\right.$, $\operatorname{Im}\left(\zeta_{\frac{\pi}{2}}(\sigma)\right)$, where $\sigma$ varies in the interval $(1,1+\varepsilon)$, makes infinitely many turns around the origin. 
Proof. From Lemma 4 (see the point iv)), we deduce that $A_{\zeta_{\frac{\pi}{2}}}(\sigma):(1, \infty) \mapsto$ $(0, \infty)$, defined as $A_{\zeta_{\frac{\pi}{2}}}(\sigma)=\sum_{k>1}\left|\operatorname{Arg}\left(1-i p_{k}^{-\sigma}\right)\right|$, is a decreasing function and it is also bijective. Likewise, by Lemma 4, point iii), we have that $\lim _{\sigma \rightarrow 1^{+}} A_{\zeta_{\frac{\pi}{2}}}(\sigma)=\infty$. In this way, in virtue of

$$
\operatorname{Arg}\left(\zeta_{\frac{\pi}{2}}(\sigma)\right)=-\sum_{k \geq 1} \operatorname{Arg}\left(1-i p_{k}^{-\sigma}\right)=\sum_{k \geq 1} \arctan \left(p_{k}^{-\sigma}\right)(\bmod (-\pi, \pi]),
$$

it is clear that, fixed $\theta \in(-\pi, \pi]$, the equality $\operatorname{Arg}\left(\zeta_{\frac{\pi}{2}}(\sigma)\right)=\theta$ is attained for infinitely many values in $(1,1+\varepsilon)$, with $\varepsilon>0$, and the curve originated from the points $\left(\operatorname{Re}\left(\zeta_{\frac{\pi}{2}}(\sigma)\right), \operatorname{Im}\left(\zeta_{\frac{\pi}{2}}(\sigma)\right)\right)$, with $\sigma \in(1,1+\varepsilon)$, makes infinitely many turns around the origin.

Remark 6 . In view of Lemma 2 , it can be easily seen that the result above is also valid for other cases such as $\zeta_{\mathbf{x}}(\sigma)$ where $\mathbf{x} \in \mathbb{R}^{\infty}$ is a vector whose components are all $\pm \frac{\pi}{2}$ except at most a finite amount of them.

Now, given $\theta \in[0, \pi]$ and $\mathbf{x} \in \mathbb{R}^{\infty}$, define

$$
\sigma_{\theta}^{\mathbf{x}}:=\sup \left\{\sigma>1:\left|\operatorname{Arg}\left(\zeta_{\mathbf{x}}(\sigma+i t)\right)\right|=\theta \text { for some } \sigma+i t \in U\right\} .
$$

In virtue of Lemma 5 and [9, Theorem 18] it is now clear that $\sigma_{\theta}^{\mathbf{x}}$ is well defined, as the set $\left\{\sigma>1:\left|\operatorname{Arg}\left(\zeta_{\mathbf{x}}(\sigma+i t)\right)\right|=\theta\right.$ for some $\left.\sigma+i t \in \mathbb{C}\right\}$ is not empty for any $\theta \in[0, \pi]$. Moreover, it coincides with

$$
\left.\sigma_{\theta}^{\mathbf{x}}=\sup \left\{\sigma>1: A_{\zeta_{\mathbf{x}}}(\sigma+i t)\right)=\theta \text { for some } \sigma+i t \in U\right\} .
$$

In this respect, we next deduce from Lemma 4 that $\sigma_{\theta}^{\mathbf{x}}$ is bounded above by

$$
\sigma_{\theta}^{\frac{\pi}{2}}:=\sup \left\{\sigma>1:\left|\operatorname{Arg}\left(\zeta_{\frac{\pi}{2}}(\sigma+i t)\right)\right|=\theta \text { for some } \sigma+i t \in U\right\}
$$

or

$$
\sigma_{\theta}^{-\frac{\pi}{2}}:=\sup \left\{\sigma>1:\left|\operatorname{Arg}\left(\zeta_{-\frac{\pi}{2}}(\sigma+i t)\right)\right|=\theta \text { for some } \sigma+i t \in U\right\}
$$

(in view of the Euler products, there is no doubt about the symmetry between $\operatorname{Arg}\left(\zeta_{\frac{\pi}{2}}(s)\right)$ and $\operatorname{Arg}\left(\zeta_{-\frac{\pi}{2}}(s)\right)$, which yields that $\left.\sigma_{\theta}^{\frac{\pi}{2}}=\sigma_{\theta}^{-\frac{\pi}{2}}\right)$.

Lemma 7. Let $\theta \in[0, \pi]$ and $\mathbf{x} \in \mathbb{R}^{\infty}$. Then $\sigma_{\theta}^{\mathbf{x}} \leq \sigma_{\theta}^{\frac{\pi}{2}}=\sigma_{\theta}^{-\frac{\pi}{2}}$.

Proof. Given $\sigma>1$ and $\mathbf{x} \in \mathbb{R}^{\infty}$, Lemma 4 assures that

$$
A_{\zeta_{\mathrm{x}}}(\sigma+i t) \leq A_{\zeta_{\frac{\pi}{2}}}(\sigma)=A_{\zeta_{-\frac{\pi}{2}}}(\sigma) .
$$

Moreover, in view of (5), it is satisfied

$$
\sigma_{\theta}^{\frac{\pi}{2}}=\sup \left\{\sigma>1: A_{\zeta \frac{\pi}{2}}(\sigma+i t)=\theta \text { for some } \sigma+i t \in U\right\} .
$$

Now, given $\mathbf{x}_{0} \in \mathbb{R}^{\infty}$, suppose by reductio ad absurdum that $\sigma_{\theta}^{\mathbf{x}_{0}}>\sigma_{\theta}^{\frac{\pi}{2}}$. This yields the existence of $s_{0}=\sigma_{0}+i t_{0} \in U$, with $\sigma_{0}>\sigma_{\theta}^{\frac{\pi}{2}}$, satisfying the equality $A_{\zeta_{\mathrm{x}_{0}}}\left(\sigma_{0}+i t_{0}\right)=\theta$. Hence 


$$
\theta=A_{\zeta_{\mathbf{x}_{0}}}\left(\sigma_{0}+i t_{0}\right) \leq A_{\zeta_{\frac{\pi}{2}}}\left(\sigma_{0}\right)<\theta,
$$

which is a contradiction.

In particular, this result yields the existence of a real number $\sigma_{\pi}>1$ such that the images of any function equivalent to the Riemann zeta function on the half-plane $\left\{s \in \mathbb{C}: \operatorname{Re} s>\sigma_{\pi}\right\}$ cannot take negative real values.

For the following result, fixed $\theta \in[0, \pi]$, take the notation

$$
\sigma_{\theta}:=\sup \{\sigma>1:|\operatorname{Arg}(\zeta(\sigma+i t))|=\theta \text { for some } \sigma+i t \in U\} .
$$

Proposition 8. Let $\theta \in[0, \pi]$ and $\mathbf{x} \in \mathbb{R}^{\infty}$. Then

(i) $\left|\operatorname{Arg}\left(\zeta_{\mathbf{x}}(s)\right)\right|<\theta$ for any $s=\sigma+i t \in U$ with $\sigma>\sigma_{\theta}$;

(ii) $\sigma_{\theta} \leq \sup \left\{\sigma>1: \sum_{k \geq 1} \arctan \left(p_{k}^{-\sigma}\right)=\theta\right\}$.

Proof. (i) By definition of $\sigma_{\theta}$, it is plain that

$$
|\operatorname{Arg}(\zeta(s))|<\theta \forall s=\sigma+i t \in U: \sigma>\sigma_{\theta} .
$$

Now, the result follows from [9, Theorem 18], as

$$
\bigcup_{\sigma>\sigma_{\theta}} \operatorname{Img}(\zeta(\sigma+i t))=\bigcup_{\sigma>\sigma_{\theta}} \operatorname{Img}\left(\zeta_{\mathbf{x}}(\sigma+i t)\right) .
$$

(ii) We already know that

$$
\sigma_{\theta}=\sup \{\sigma>1:|\operatorname{Arg}(\zeta(\sigma+i t))|=\theta\}=\sup \left\{\sigma>1: A_{\zeta}(\sigma+i t)=\theta\right\} .
$$

By Lemma 4 , it is accomplished that

$$
A_{\zeta_{\frac{\pi}{2}}}(s)=A_{\zeta_{-\frac{\pi}{2}}}(s)=\sum_{k \geq 1}\left|\arctan \left(p_{k}^{-\sigma}\right)\right|=\sum_{k \geq 1} \arctan \left(p_{k}^{-\sigma}\right) .
$$

Finally, the result follows from Lemma 7.

We next focus our attention on the vortex-like behaviour of the Riemann zeta function, and of every function that is equivalent to it. For this reason, we first show the following two preliminary results.

Proposition 9. Let $\mathbf{x} \in \mathbb{R}^{\infty}, \sigma_{0}>1, \theta \in(-\pi, \pi]$ and $n \in \mathbb{N}$. Then there exists a real number $\rho_{n}$ with $1<\rho_{n}<\sigma_{0}$ such that $\operatorname{Arg}\left(\zeta_{\mathbf{x}}(s)\right)=\theta$ is satisfied for at least $n$ distinct values in the vertical strip $\left\{s \in \mathbb{C}: \rho_{n}<\operatorname{Re} s<\sigma_{0}\right\}$.

Proof. Given $\varepsilon>0$, we already know from Lemma 5 that the parametrized curve originated from the points $\left(\operatorname{Re}\left(\zeta_{\frac{\pi}{2}}(\sigma)\right), \operatorname{Im}\left(\zeta_{\frac{\pi}{2}}(\sigma)\right)\right)$, with $\sigma \in(1,1+\varepsilon)$, makes infinitely many turns around the origin. In particular, given $\sigma_{0}>1$, $\theta \in(-\pi, \pi]$ and $n \in \mathbb{N}$, there exists a real number $\rho_{n}$ with $1<\rho_{n}<\sigma_{0}$ such that $\operatorname{Arg}\left(\zeta_{\frac{\pi}{2}}(\sigma)\right)=\theta$ is satisfied for at least $n$ distinct values in $\left(\rho_{n}, \sigma_{0}\right)$. Now, given $\mathbf{x} \in \mathbb{R}^{\infty}$, we deduce from [9, Theorem 18] that

$$
\bigcup_{\sigma \in\left(\rho_{n}, \sigma_{0}\right)} \operatorname{Img}\left(\zeta_{\mathbf{x}}(\sigma+i t)\right)=\bigcup_{\sigma \in\left(\rho_{n}, \sigma_{0}\right)} \operatorname{Img}\left(\zeta_{\frac{\pi}{2}}(\sigma+i t)\right) .
$$


Then $\operatorname{Arg}\left(\zeta_{\mathbf{x}}(s)\right)=\theta$ is satisfied for at least $n$ distinct values in the vertical strip $\left\{s \in \mathbb{C}: \rho_{n}<\operatorname{Re} s<\sigma_{0}\right\}$ and the result follows.

Proposition 10. Let $\mathbf{x} \in \mathbb{R}^{\infty}, \sigma_{0}>1$ and $\theta \in(-\pi, \pi]$. Then $\operatorname{Arg}\left(\zeta_{\mathbf{x}}(s)\right)=\theta$ is satisfied for infinitely many values of $s \in \mathbb{C}$ with $1<\operatorname{Re} s<\sigma_{0}$.

Proof. Fixed $\varepsilon>0$, we will again use the fact that the curve originated from the images $\zeta_{\frac{\pi}{2}}(\sigma)$, with $\sigma \in(1,1+\varepsilon)$, makes infinitely many turns around the origin (Lemma 5). In this way, given $\theta \in(-\pi, \pi]$, there exists a set $C \subset(1,1+\varepsilon)$ consisting of infinitely many isolated values $\sigma$ satisfying $\operatorname{Arg}\left(\zeta_{\frac{\pi}{2}}(\sigma)\right)=\theta$. Now, given $\sigma_{0} \in C$, by continuity choose $\delta_{\sigma_{0}}>0$ such that the parametrized curve traced by the points $\left(\operatorname{Re}\left(\zeta_{\frac{\pi}{2}}(\sigma)\right), \operatorname{Im}\left(\zeta_{\frac{\pi}{2}}(\sigma)\right)\right)$, with $\sigma \in\left(\sigma_{0}-\delta_{\sigma_{0}}, \sigma_{0}+\delta_{\sigma_{0}}\right)$, makes one turn around the origin. Thus we deduce from [9, Theorem 18] that

$$
\bigcup_{\sigma \in\left(\sigma_{0}-\delta_{\sigma_{0}}, \sigma_{0}+\delta_{\sigma_{0}}\right)} \operatorname{Img}\left(\zeta_{\mathbf{x}}(\sigma+i t)\right)=\bigcup_{\sigma \in\left(\sigma_{0}-\delta_{\sigma_{0}}, \sigma_{0}+\delta_{\sigma_{0}}\right)} \operatorname{Img}\left(\zeta_{\frac{\pi}{2}}(\sigma+i t)\right) .
$$

Hence $\operatorname{Arg}\left(\zeta_{\mathbf{x}}(s)\right)=\theta$ is satisfied at least once in the vertical strip $E_{\sigma_{0}}:=\{s \in$ $\left.\mathbb{C}: \sigma_{0}-\delta_{\sigma_{0}}<\operatorname{Re} s<\sigma_{0}+\delta_{\sigma_{0}}\right\}$. Finally, by varying $\sigma_{0}$ in the set $C$, the result follows.

Finally, by using [7, Theorem 2], we next improve the results above in the following sense.

Theorem 11. Let $\mathbf{x} \in \mathbb{R}^{\infty}, \sigma_{0}>1$ and $n \in \mathbb{N}$. Then there exists a relatively dense set of real numbers $\left\{t_{n, m}\right\}_{m \geq 1}$ such that, for each $m=1,2, \ldots$, the parametrized curve traced by the points $\left(\operatorname{Re}\left(\zeta_{\mathbf{x}}\left(\sigma+i t_{n, m}\right)\right), \operatorname{Im}\left(\zeta_{\mathbf{x}}\left(\sigma+i t_{n, m}\right)\right)\right)$, where $\sigma \in\left(1, \sigma_{0}\right)$, makes at least $n$ turns around the origin.

Proof. It is worth noting that [7, Corollary 5] assures the existence of an increasing unbounded sequence $\left\{\tau_{j}\right\}_{j \geq 1}$ of positive numbers such that the sequence of functions $\left\{\zeta_{\mathbf{x}}\left(s+i \tau_{j}\right)\right\}_{j \geq 1}$ converges uniformly to $\zeta_{\frac{\pi}{2}}(s)$ on every reduced strip of $U=\{s \in \mathbb{C}: \operatorname{Re} s>1\}$. In fact, given $\sigma_{0}>1, \varepsilon>0$ and a reduced strip $U_{\varepsilon}=\left\{s \in \mathbb{C}: 1+\varepsilon<\operatorname{Re} s<\sigma_{0}\right\} \subset U$, also by [7, Corollary 5] there exists a relatively dense set of real numbers $\left\{t_{j}\right\}_{j \geq 1}$ such that

$$
\left|\zeta_{\mathbf{x}}\left(s+i t_{j}\right)-\zeta_{\frac{\pi}{2}}(s)\right|<\varepsilon \text { for any } s \in U_{\varepsilon} .
$$

In particular, we have

$$
\left|\zeta_{\mathbf{x}}\left(\sigma+i t_{j}\right)-\zeta_{\frac{\pi}{2}}(\sigma)\right|<\varepsilon \text { for any } \sigma \in\left(1+\varepsilon, \sigma_{0}\right) .
$$

Equivalently,

$$
\zeta_{\mathbf{x}}\left(\sigma+i t_{j}\right)=\zeta_{\frac{\pi}{2}}(\sigma)+\delta_{j}, \text { with }\left|\delta_{j}\right|<\varepsilon, \sigma \in\left(1+\varepsilon, \sigma_{0}\right) .
$$

Likewise, given $\theta \in(-\pi, \pi]$, we deduce from $\operatorname{Lemma} 5$ that $\operatorname{Arg}\left(\zeta_{\frac{\pi}{2}}(\sigma)\right)=\theta$ is attained for a set $C$ of infinitely many values $\sigma$ in $\left(1, \sigma_{0}\right)$. In fact, given $n \in \mathbb{N}$, there exists $\varepsilon$ sufficiently small such that $\operatorname{Arg}\left(\zeta_{\frac{\pi}{2}}(\sigma)\right)=\theta$ is satisfied for at least $n+1$ distinct values in $\left(1+\varepsilon, \sigma_{0}\right)$, and the curve originated from the points $\left(\operatorname{Re}\left(\zeta_{\frac{\pi}{2}}(\sigma)\right), \operatorname{Im}\left(\zeta_{\frac{\pi}{2}}(\sigma)\right)\right)$, with $\sigma \in\left(1+\varepsilon, \sigma_{0}\right)$, makes at least $n+1$ turns 
around the origin. In terms of the decreasing function $A_{\zeta \frac{\pi}{2}}\left(\sigma_{0}\right)$ (see Lemma 4, point iv)), this means that

$$
A_{\zeta_{\frac{\pi}{2}}}(1+\varepsilon)-A_{\zeta_{\frac{\pi}{2}}}\left(\sigma_{0}\right)>2 \pi n
$$

Thus, by taking $\varepsilon$ sufficiently small and taking into account that $\zeta_{\mathbf{x}}(s) \neq 0$ for any $s \in U$, we deduce from (6) and (7) the existence of a sequence $\left\{t_{n, m}\right\}_{m>1}$ of real numbers satisfying the following property: For each $m=1,2, \ldots$, the equality $\operatorname{Arg}\left(\zeta_{\mathbf{x}}\left(\sigma+i t_{n, m}\right)\right)=\theta$ is satisfied for at least $n$ values $\sigma$ in $(1+$ $\left.\varepsilon, \sigma_{0}\right)$ (near the points $C \cap\left(1+\varepsilon, \sigma_{0}\right)$ ) and the curve traced by the points $\left(\operatorname{Re}\left(\zeta_{\mathbf{x}}\left(\sigma+i t_{n, m}\right)\right), \operatorname{Im}\left(\zeta_{\mathbf{x}}\left(\sigma+i t_{n, m}\right)\right)\right)$, with $\sigma \in\left(1+\varepsilon, \sigma_{0}\right)$, makes at least $n$ turns around the origin. Indeed, if $\sigma_{1} \in C \cap\left(1+\varepsilon, \sigma_{0}\right)$ and $\theta \in(-\pi, \pi)$ then $\operatorname{Arg}\left(\zeta_{\frac{\pi}{2}}\left(\sigma_{1}\right)\right)=\theta, \operatorname{Arg}\left(\zeta_{\frac{\pi}{2}}\left(\sigma_{1}-\rho\right)\right)>\theta$ and $\operatorname{Arg}\left(\zeta_{\frac{\pi}{2}}\left(\sigma_{1}+\rho\right)\right)<\theta$ for values of $\rho>0$ sufficiently small in an interval $\left(0, a_{\sigma_{1}}\right)$. Now, fixed $\varepsilon>0$, thanks to $(7)$ we assure the existence of $\rho_{\varepsilon} \in\left(0, a_{\sigma_{1}}\right)$ satisfying $\operatorname{Arg}\left(\zeta_{\mathbf{x}}\left(\sigma-\rho_{\varepsilon}+i t_{n, m}\right)\right)>\theta$ and $\operatorname{Arg}\left(\zeta_{\mathbf{x}}\left(\sigma+\rho_{\varepsilon}+i t_{n, m}\right)\right)<\theta$, which yields by continuity that $\operatorname{Arg}\left(\zeta_{\mathbf{x}}(\sigma+\right.$ $\left.\left.i t_{n, m}\right)\right)=\theta$ for some $\sigma \in\left(\sigma_{1}-\rho_{\varepsilon}, \sigma_{1}+\rho_{\varepsilon}\right)$.

In particular, the result above can be particularized for the significant case of the Riemann zeta function.

Corollary 12. Let $\sigma_{0}>1$ and $n \in \mathbb{N}$. Then there exists a relatively dense set of real numbers $\left\{t_{n, m}\right\}_{m \geq 1}$ such that, for each $m=1,2, \ldots$, the parametrized curve traced by the points $\left(\operatorname{Re}\left(\zeta\left(\sigma+i t_{n, m}\right)\right), \operatorname{Im}\left(\zeta\left(\sigma+i t_{n, m}\right)\right)\right)$, with $\sigma \in$ $\left(1, \sigma_{0}\right)$, makes at least $n$ turns around the origin.

Moreover, Theorem 11 (and other results as Lemma 7 and Proposition 8) can also be immediately extended to its reciprocal sum (and all exponential sums included in its equivalence class) which is expressed as a Dirichlet series over the Möbius function $\mu(n)$ in the following terms (see $[12$, p.3]):

$$
\frac{1}{\zeta(s)}=\sum_{n \geq 1} \frac{\mu(n)}{n^{s}}=\prod_{k=1}^{\infty}\left(1-p_{k}^{-s}\right), \operatorname{Re} s>1 .
$$

\section{Acknowledgements}

The first author was supported by PGC2018-097960-B-C22 (MCIU/AEI/ ERDF, UE).

Funding Open Access funding provided thanks to the CRUE-CSIC agreement with Springer Nature. 
Data Availability Statement Data sharing not applicable to this article as no datasets were generated or analysed during the current study.

\section{Declarations}

Conflict of interest The authors declare that they have no conflict of interest.

Open Access. This article is licensed under a Creative Commons Attribution 4.0 International License, which permits use, sharing, adaptation, distribution and reproduction in any medium or format, as long as you give appropriate credit to the original author(s) and the source, provide a link to the Creative Commons licence, and indicate if changes were made. The images or other third party material in this article are included in the article's Creative Commons licence, unless indicated otherwise in a credit line to the material. If material is not included in the article's Creative Commons licence and your intended use is not permitted by statutory regulation or exceeds the permitted use, you will need to obtain permission directly from the copyright holder. To view a copy of this licence, visit http://creativecommons.org/ licenses/by $/ 4.0 /$.

\section{References}

[1] Apostol, T.M.: Modular Functions and Dirichlet Series in Number Theory. Springer, New York (1990)

[2] Bohr, H.: Zür Theorie der allgemeinen Dirichletschen Reihen. Math. Ann. 79, 136-156 (1919)

[3] Borwein, P., Choi, S., Rooney, B., Weirathmueller, A. (eds.): The Riemann Hypothesis. CMS Books in Mathematics. A Resource for the Afficionado and Virtuoso Alike. Springer, New York (2008)

[4] Lehman, R.S.: On Liouville's function. Math. Comput. 14, 311-320 (1960)

[5] Mitrinović, D.S., Pečarić, J.E., Fink, A.M.: Classical and New Inequalities in Analysis. Kluwer Academics Publishers, Dordrecht (1993)

[6] Righetti, M.: On Bohr's equivalence theorem. J. Math. Anal. Appl., 445 (1) (2017) 650-654 (Corrigendum, ibid. 449 (2017) 939-940)

[7] Sepulcre, J.M., Vidal, T.: Almost periodic functions in terms of Bohr's equivalence relation. Ramanujan J., 46 (1) (2018) 245-267 (Corrigendum, ibid, 48 (3) (2019) 685-690)

[8] Sepulcre, J.M., Vidal, T.: Bohr's equivalence relation in the space of Besicovitch almost periodic functions. Ramanujan J. 49(3), 625-639 (2019)

[9] Sepulcre, J.M., Vidal, T.: A generalization of Bohr's equivalence theorem. Complex Anal. Oper. Theory 13(4), 1975-1988 (2019)

[10] Sepulcre, J.M., Vidal, T.: Sets of values of equivalent almost periodic functions. Ramanujan J. 56(1), 87-102 (2021)

[11] Spira, R.: Sets of values of general Dirichlet series. Duke Math. J. 35(1), 79-82 (1968) 
[12] Titchmarsh, E.C.: The Theory of the Riemann Zeta-Function, 2nd edn. Oxford Science publication (1986)

J. M. Sepulcre

Departamento de Matemáticas

Universidad de Alicante

03080 Alicante

Spain

e-mail: JM.Sepulcre@ua.es

T. Vidal

Universidad Nacional de Educación a Distancia (UNED)

CL. Candalix s/n

03202 Elche, Alicante

Spain

e-mail: tmvg@alu.ua.es

Received: May 8, 2021.

Accepted: November 25, 2021.

Publisher's Note Springer Nature remains neutral with regard to jurisdictional claims in published maps and institutional affiliations. 